\title{
Author Index Vol. 70, 1996
}

The authors of the Abstracts of the 7th European Workshop on Neo-natology are indexed separately in Vol. 70, No. 3, pp. 191-192, 1996

Aasen,S.E. 249 Abraham, C.S. 296 Abrams,R.M. 155 Alarcon, P.A. de 218 Allen, J.W. 289

Anderson, C.A. 265 Anderson-Morris, J. 349 Arduini,D. 84 Ayusawa, M. 15

Ballèvre, L. 1 Bartmann, P. 128 Bauer, R. 155 Benders, M.J.N.L. 141 Berseth,C.L. 265 Besnier, S. 29 Bethmann, O. de 328 Beyaert,C. 359 Blum,D. 304 Bracci, R. 21 Bratlid,D. 249

Bucher,H.-U. 91 Buonocore, G. 21 Burdge,G.C. 108 Burmeister, L.F. 218 Byford,A.M. 199 Caccamo, M.L. 322 Capponi, A. 84 Catella,A,M. 21 Christensen, T. 249 Cieuta,C. 246 Claris, O. 29 Clercx,A. 304 Cook,R.T. 218 Corazza, F. 304

D’Antuono, C. 235 Daffos,F. 193 Dalkin,A.C. 199 Deguchi, Y. 35 Delacourt, C. 246 Devaskar, U.P. 101 Dietrich, P. 271 Dorrepaal, C.A. 141

Endo, A. 15

Fernández-Tomé, M.d.C. 235 Feuer, P. 52 Figueroa, H.R. 165 Fondu,P. 304 Forestier, F. 193 Frank, L. 116 Friedrich,W. 75

Gatti, L. 322 Georgieff, M.K. 218 Gerhardt, K.J. 155 Gianotti, G.A. 322 Gilrain,J.T. 199

Govindrajan, R. 101 Gozal,D. 280 Gozal,Y.M. 280 Griese,M. 271 Guarneri, D. 322 Guignard, J.-P. 1

Halpern, S. 213 Hammerman, C. 69 Hansen, T.W.R. 289 Haouzi,P. 359 Harada, K. 15

Hardjowijono, R. 141 Haufe,M. 75 Hausman, D.B. 41 Hausman, GJ. 41 Hengartner, M. 91

Hervias, M.C. 165 Heyman, S. 101 Hiraoka, M. 35 Hori,C. 35

Innanen, V. 213

Jacquemard, F. 193 Jarreau, P.-H. 246 Jenni,O.G. 91 Johnsson, A. 249 Jones, A.C. 108

Kahane,V.L. 235 Kaplan, M. 69 Kastler,B. 328 Kauffman, R.E. 135 Kawamitsu, T. 35 Keel,M. 91 Kimura, K. 35 Kling,R. 75 Konishi,Y. 35 Kovács,J. 296 Krishnan, M. 135 Krishnan, R. 135 Kumar, P. 135 Kurth,C.D. 52 Kusaka,Y. 35

Lapillonne, A. 29 Law,R.M.T. 213 Lehrnbecher, T. 206 Lindberg, T. 311 Lopez, C. 229 Lopez, M. 229 Lowe,L.S. 218 Lucena, J. 135

Mao,Y.-L. 60 Marchal,F. 359 Mardens, Y. 304 Marini, A. 322 Martin, R.J. 41 Martins, R.F. 213

Martius, J. 206 Masaoka, N. 15 Matherne, G.P. 199 Matsuda,M. 349 Meléndez, E. 229

Mello,D.E.de 101 Minato, M. 15 Mirlesse,V. 193 Molnár,D. 296 Monitto,C.L. 52 Monsert-

Couchard, M. 328

Neumeister, B. 128 Nuckton,T.J. 280

O’Rourke, M. 52 Ohlsson,A. 213

Pascual,R. 165 Peters, A. J.M. 155 Peters, C. 218 Picaud,J.C. 29 Postle,A.D. 108 Potz,C. 271

Poveda,J.-D. 246 Price, L.T. 116

Reich, H. 213 Reyes, J.L. 229 Rinaldi,G. 21 Rinaldo,D. 84 Rizzo,G. 84 Romanini, C. 84

Roos, T. 206 Rutsch,P. 206

Salle,B.L. 29 Sato,K. 15 
Scheinmann, P. 246 Schmalisch, G. 75 Schrod, L. 206 Schultz,K. 296 Schwab, M. 155 Serenius, F. 311 Shankaran, S. 135 Shaw,L. 52 Shimada, M. 15 Siebenthal, K. von 91 Solhaug,MJ. 1 Sosenko, I.R.S. 101 Speziale, E.H.S. 235 Sterin-Speziale, N.B. 235 Stevenson, D.K. 69 Sudo,M. 35

Takada,M. 15 Takahashi, S. 15 Temesvári, P. 296 Törnhage, C.-J. 311 Torres, J.E. 280 Toti,P. 21

Tso, M.-Y.W. 60 Tsukahara, H. 35

UshaRaj,J. 349 Uvnús-Moberg, K. 311

Van Bel, F. 141 Van De Bor, M. 141 Vreman,H.J. 69

Wang,T. 339 Warner, J.A. 108 Warner, J.O. 108 Wauer,R.R. 75 Westerburg, B. 271 Whitney, Ph.L. 116 Widness, J.A. 218 Woerndle, S. 128 Wolf,M. 91

Xu, R.-J. 60, 339

Zeeuwe, P.E.M. 141

366

AuthorlndexVol. 70,1996 